\title{
Latent heat of nuclear matter
}

\author{
Arianna Carbone and Artur Polls \\ Departament d'Estructura i Constituents de la Matèria and Institut de Ciències del Cosmos, \\ Universitat de Barcelona, Avda. Diagonal 647, E-08028 Barcelona, Spain
}

\author{
Arnau Rios \\ Department of Physics, University of Surrey, \\ Guildford, Surrey, GU2 7XH, United Kingdom \\ Isaac Vidaña \\ Centro de Física Computacional, Department of Physics, \\ University of Coimbra, PT-3004-516 Coimbra, Portugal
}

(Dated: January 15, 2011)

\begin{abstract}
We study the latent heat of the liquid-gas phase transition in symmetric nuclear matter using self-consistent mean-field calculations with a few Skyrme forces. The temperature dependence of the latent heat is rather independent of the mean-field parametrization and can be characterized by a few parameters. At low temperatures, the latent heat tends to the saturation energy. Near the critical point, the latent heat goes to zero with a well-determined mean-field critical exponent. A maximum value of the latent heat in the range $l \sim 25-30 \mathrm{MeV}$ is found at intermediate temperatures, which might have experimental relevance. All these features can be explained from very basic principles.
\end{abstract}

PACS numbers: 21.60.Jz, 21.65.-f, 64.60.F- 


\section{INTRODUCTION}

The study of the liquid-gas phase transition in homogeneous symmetric nuclear matter provides interesting links between statistical mechanics, quantum physics and nuclear dynamics [1-3]. The liquid-gas phase transition picture can, in principle, be applied to nuclear systems [4]. The nucleon-nucleon (NN) interaction has a qualitative similar structure to the inter-atomic one: it is repulsive at short relative distances and attractive at intermediate and long distances [5]. At zero temperature, this structure drives the particles to stay at a given distance from each other, thus leading to a structured liquid phase. As temperature increases, thermal fluctuations overcome the interaction effects and the system tends to gasify, thus causing a phase transition [6].

The only way to study thermodynamical properties of nuclear systems on Earth is via intermediate and heavy ion collisions $[1,4]$, which unfortunately are particularly fast $(\sim$ $10^{-21} \mathrm{~s}$ ) processes and very difficult to model. Unlike in other branches of physics, one cannot define or prepare samples for a thermodynamical experiment. Furthermore, in a collision the state of the system changes continuously in time, and it is difficult to establish whether the system has actually reached a state of equilibrium or not $[7,8]$. Finally, the fact that we are dealing with finite nuclear matter causes additional difficulties on the thermodynamical interpretation of the results [9].

Experimentally, the challenge lies in being able to control the equilibrium, i.e. in determining the state variables of the system, like the temperature, the pressure or the density of the system $[10,11]$. Moreover, one also needs to find suitable observables that help to identify the liquid-gas phase transition. Candidates include, among others, the critical behavior of fragment partitions [12-14], energy fluctuations on an event by event basis [15], nuclear calorimetry $[16,17]$ and bimodality of the largest fragment distribution $[18,19]$. The evidence gathered with these different experimental techniques points towards the existence of a liquid-gas phase transition for nuclear systems at densities below the empirical saturation density, $\rho_{0}=0.16 \mathrm{fm}^{-3}$, and temperatures around $5-10 \mathrm{MeV}$.

From the theoretical side, symmetric nuclear matter at finite temperature provides a first qualitative picture of the thermodynamics of nuclear systems and, particularly, of the liquid-gas phase transition [2,3]. Because of its relative simplicity, nuclear matter has been the subject of numerous investigations. From a microscopic perspective, different 
many-body techniques (including Dirac-Brueckner [20] and Brueckner-Hartree-Fock [21], Self-Consistent Green's functions [22, 23] or variational calculations [24]) have been used to compute the thermodynamical properties of nuclear matter starting from basic nucleonic degrees of freedom and phase-shift equivalent realistic NN interactions. So far, very different predictions for critical properties have been obtained using different approaches or even different interactions within the same approach [22]. A more efficient way to get insight in the thermal behaviour of nuclear matter is by means of effective interactions in the framework of a Hartree-Fock approximation, either relativistic [25] or non-relativistic [26, 27]. Mean-field calculations are much faster to implement numerically and give access to a wider range of phenomena (critical exponents, for instance). Moreover, the mean-field parametrizations are directly connected to nuclear structure [28]. Recently, one of us has studied systematically the dependence on the effective interaction of the liquid-gas phase transition of nuclear matter with Skyrme and Gogny mean-fields [29].

In the context of homogeneous nuclear matter, one generally assumes that thermodynamical equilibrium takes place between two infinite pieces of nuclear matter, one of them belonging to the liquid phase and the other to a gaseous state. This is quite a crude approximation that can only provide an average, qualitative understanding of the thermal properties of the liquid-gas phase transition. As a matter of fact, one should consider that the lowdensity phase of dense nuclear matter is not necessarily homogeneous [30]. Its description should allow also for the formation of light clusters of nucleons, including deuterons, tritons, helions $\left({ }^{3} \mathrm{He}\right)$ or $\alpha$-particles [31-34]. Moreover, finite size and Coulomb effects are very important to determine the thermal properties and the critical behavior of finite fermionic systems [35].

Very little attention has been paid so far to the study of the latent heat of nuclear matter, which is the main goal of this work. This is a very interesting quantity, since it can provide a further characterization of the liquid-gas phase transition, and it can be potentially extracted both from experiments and theory. Experimentally, the latent heat might be, in principle, read out from the length of the plateau in the caloric curve (i.e. in the temperature vs. excitation energy curve). This would suggest values of $l \sim 4-8 \mathrm{MeV}[16,17,36]$. Note, however, that this plateau can also be explained in other terms, rather than in a phase transition picture [37-39]. Alternatively, the latent heat can also be obtained from the heat capacity versus excitation energy, as shown in Ref. [15], in which case $l \sim 2-4 \mathrm{MeV}$. 
Recently, bimodality in the charge distribution of the heaviest fragments has been used to extract a latent heat value of $l \sim 8 \mathrm{MeV}$ [19]. In any case, one has to be aware of the fact that, for the latent heat to be defined, the reaction employed to extract it should not be an isentropic process [40].

In the case of infinite nuclear matter, the latent heat per particle accounts for the amount of heat needed to take a nucleon from the liquid to the gas phase. In this work, we want to study this quantity and provide estimates for its typical values in nuclear matter. We will, in particular, describe its basic temperature dependence with different Skyrme mean-field parametrizations. We also want to link the behavior of this quantity to the properties of the underlying NN interaction. Ultimately, our aim is to understand the behavior of the latent heat from basic quantum statistical mechanics arguments.

In the next section, we discuss very briefly the formalism needed for the calculation of the latent heat within the Skyrme-Hartree-Fock approximation. After that, in Section III, we present and discuss the self-consistent results, which is followed by a section devoted to the analysis of the low temperature and critical behavior of the latent heat. A summary and final conclusions are presented in the last section.

\section{FORMALISM}

To describe efficiently finite nuclei and nuclear matter at the same time, one generally relies on phenomenological interactions, adequate for the Hartee-Fock (HF) approximation. The effective forces used in the following are of the Skyrme type. They were introduced in the 1950's [41, 42], and have been intensively used in the literature [43]. Alternatively, one can obtain equivalent results by formulating the problem in terms of density functionals $[28,44]$.

In this work we deal with a Skyrme effective interaction:

$$
\begin{aligned}
\hat{v}_{i j} & =t_{0}\left(1+x_{0} P_{\sigma}\right) \delta(\vec{r})+\frac{1}{2} t_{1}\left(1+x_{1} P_{\sigma}\right)\left[\delta(\vec{r}) \vec{k}^{2}+\overleftarrow{k}^{2} \delta(\vec{r})\right] \\
& +t_{2}\left(1+x_{2} P_{\sigma}\right) \overleftarrow{k} \delta(\vec{r}) \vec{k}+\frac{1}{6} t_{3}\left(1+x_{3} P_{\sigma}\right)[\rho(\vec{R})]^{\alpha} \delta(\vec{r})
\end{aligned}
$$

where $\vec{R}=\left(\overrightarrow{r_{i}}+\overrightarrow{r_{j}}\right) / 2, \vec{r}=\vec{r}_{i}-\overrightarrow{r_{j}}, \vec{k}=\left(\vec{\nabla}_{i}-\vec{\nabla}_{j}\right) / 2 i$ is acting on the right; $\overleftarrow{k}=-\left(\overleftarrow{\nabla}_{i}-\overleftarrow{\nabla}_{j}\right) / 2 i$, acting on the left; $P_{\sigma}=\left(1+\overrightarrow{\sigma_{1}} \cdot \overrightarrow{\sigma_{2}}\right) / 2$ is the spin-exchange operator ( $\vec{\sigma}$ are Pauli matrices; ), and $\rho=N / \Omega$, the total baryonic density. The parameters 
$t_{0}, t_{1}, t_{2}, t_{3}, x_{0}, x_{1}, x_{2}, x_{3}, \alpha$ are numerical constants fitted to reproduce, in general, the saturation properties of nuclear matter and structure properties of closed shell nuclei [43]. Three-body interactions are effectively accounted for by the last density-dependent term. Mean-field calculations with effective Skyrme forces reproduce in a satisfactory way the structure of a wide range of nuclei [28].

Due to the translational invariance of uniform nuclear matter, single-particle (s.p.) states are appropriately described by plane waves, and the relevant quantum numbers are the s.p. momentum, $\vec{k}$, as well as the spin and isospin projections. At finite temperature within the Hartree-Fock approximation, momentum states are occupied according to the Fermi-Dirac distribution,

$$
n(\vec{k}, T)=\frac{1}{1+e^{(\varepsilon(\vec{k})-\mu) / T}},
$$

where $T$ is the temperature of the system; $\mu$, its chemical potential, and $\varepsilon(\vec{k})$, the s.p. energy. Including the rearrangement term arising from the density dependence of the effective interaction, the latter is written as:

$$
\varepsilon(\vec{k})=\frac{\hbar^{2} k^{2}}{2 m^{*}}+\frac{1}{16} \mathcal{T}\left(3 t_{1}+5 t_{2}+4 t_{2} x_{2}\right)+\frac{3}{4} \rho\left[t_{0}+\frac{1}{12}(\alpha+2) t_{3} \rho^{\alpha}\right],
$$

where the effective mass,

$$
\frac{m^{*}}{m}=\frac{1}{1+\frac{2 m}{\hbar^{2}} \frac{1}{16}\left(3 t_{1}+5 t_{2}+4 t_{2} x_{2}\right) \rho},
$$

and the kinetic energy density,

$$
\mathcal{T}=\frac{\nu}{(2 \pi)^{3}} \int \mathrm{d}^{3} k k^{2} n(\vec{k}, T)
$$

have been introduced. The integral of $n(\vec{k}, T)$ over the available phase space at finite temperature $T$ gives the total density, $\rho$ :

$$
\rho=\frac{\nu}{(2 \pi)^{3}} \int \mathrm{d}^{3} k n(\vec{k}, T),
$$

where $\nu$ is the degeneracy ( $\nu=4$ for spin and isospin saturated nuclear matter). This condition determines the chemical potential at a fixed external density. The calculation of the single-particle energy and the density normalization condition defines a self-consistent process. Note, however, that due to the simple structure of the Skyrme interaction, selfconsistency at the HF level is already achieved at the first iteration. 
Having fixed the chemical potential, the momentum distribution and the kinetic energy density are fixed. From these quantities, one can immediately compute the total HF energy per particle:

$$
e(\rho, T)=\frac{\hbar^{2}}{2 m^{*}} \frac{\mathcal{T}}{\rho}+\frac{3}{8} \rho\left[t_{0}+\frac{1}{6} t_{3} \rho^{\alpha}\right],
$$

and the entropy per particle:

$$
s(\rho, T)=\frac{\nu}{(2 \pi)^{3} \rho} \int d^{3} k[n(\vec{k}, T) \ln n(\vec{k}, T)+(1-n(\vec{k}, T)) \ln (1-n(\vec{k}, T))] .
$$

In turn, these give access to the free energy, $f=e-T s$, from which all the remaining thermodynamical properties can be computed. In addition to the chemical potential, $\mu$, the pressure, $P$, is also necessary to study the liquid-gas phase transition. One should take into account that this procedure is thermodynamically consistent, in the sense that the chemical potential extracted from the normalization of the density, Eq. (6), coincides with the chemical potential derived from the free energy, $\mu=f+\rho(\partial f / \partial \rho)$, provided that the rearrangement term is properly included.

The Clausius-Clapeyron formula,

$$
l=T\left(\frac{1}{\rho_{g}}-\frac{1}{\rho_{l}}\right)\left(\frac{\mathrm{d} P}{\mathrm{~d} T}\right)_{\text {coex }},
$$

gives access to the latent heat per particle $[6,45]$. This is expressed in terms of the product of the temperature, the difference between volume per particle of the two phases and the derivative of the pressure with respect to the temperature along the coexistence curve. Alternatively the latent heat can also be computed as the amount of heat that is needed, at a fixed temperature, to transfer one nucleon from the ordered (liquid) to the disordered (gas) phase. Since this process happens at constant chemical potential and pressure, the heat change only involves the difference in entropy per particle of the two phases:

$$
l=T\left(s_{g}-s_{l}\right)
$$

For nuclear matter calculations, this formula is numerically more stable than the ClausiusClapeyron one. Values of the vaporization specific latent heat for common liquids and gases are in the region of $10-5000 \mathrm{~kJ} / \mathrm{kg}$ when measured at the normal boiling point. In contrast, as we shall see in the following, nuclear matter has a maximum specific latent heat of the order of $\sim 30 \mathrm{MeV}$, i.e. $\sim 3 \times 10^{12} \mathrm{~kJ} / \mathrm{kg}$, which is orders of magnitude higher and among the 
highest in nature (an exception being the quark-gluon plasma). The origin of this extremely large value can be traced back to the strong force, which binds the nucleons tightly.

A qualitative model that justifies these relatively high values and provides insight on the temperature dependence of the latent heat can be obtained using basic ingredients. From Eq. (10), we see that the latent heat can be computed from the difference of entropies times the temperature. On the one hand, let us take a classical approximation for the entropy of the gas,

$$
s_{g}=\frac{5}{2}-\frac{\mu_{g}}{T} .
$$

This approximation should be valid because the gas coexistence density is, in general, quite low, which is also why interaction effects are neglected. On the other hand, the liquid phase is closer to saturation density and one should use the Sommerfeld expansion to compute its thermodynamical properties. The entropy in the liquid phase would then yield:

$$
s_{l}=\frac{\pi^{2}}{2} \frac{T}{\varepsilon_{0}}
$$

where $\varepsilon_{0}=\frac{\hbar^{2} k_{F}^{2}}{2 m}$. We have ignored interaction effects (which would appear in the form of an effective mass) and we take $k_{F}$ at a reference density equal to saturation density. Within this approximation, the latent heat becomes:

$$
l=-\mu(T)+\frac{5}{2} T-\frac{\pi^{2}}{2 \varepsilon_{0}} T^{2} .
$$

The chemical potential is the same for the liquid and the gas phases, due to the coexistence conditions (see Eq. (18) below). One can therefore use the Sommerfeld expansion in the liquid branch to find the corresponding chemical potential:

$$
\mu_{l}=\varepsilon_{0}\left[1-\frac{\pi^{2}}{12}\left(\frac{T}{\varepsilon_{0}}\right)^{2}\right]+U_{0} .
$$

Note that, again, we fix all densities to be at saturation and that $U_{0}$ is the attractive contribution needed so that $\mu_{l}(T=0)=e\left(\rho_{0}, T=0\right) \equiv-e_{0}$. Using Eq. (14) into Eq. (13), one finds the following expression for the latent heat:

$$
l=e_{0}+\frac{5}{2} T-\frac{5 \pi^{2}}{12 \varepsilon_{0}} T^{2}
$$

Within this very crude model, the latent heat is a quadratic function of the temperature. Loosely speaking, classical effects (i.e. those due to Eq. (11)) tend to increase the latent heat 
linearly in temperature. Thermal effects arising from the degenerate expansion compensate this term and lead to a maximum, occurring at a temperature,

$$
T_{\max }=\frac{3}{\pi^{2}} \varepsilon_{0} \sim 11 \mathrm{MeV}
$$

The maximum value of the latent heat is then given by the sum of two terms:

$$
l_{\max }=e_{0}+\frac{15}{4 \pi^{2}} \varepsilon_{0} \sim 30 \mathrm{MeV} .
$$

In addition to the term due to the saturation energy, an equally important term (in sign and size) appears as a result of the competition between classical and degeneracy effects. This increases the value of the maximum latent heat substantially, up to around $30 \mathrm{MeV}$. In the following, we will see that the predictions of this simple model are well fulfilled by Skyrme mean-field calculations of the liquid-gas phase transition.

\section{RESULTS}

The liquid-gas phase coexistence and the critical properties of nuclear matter studied in a mean-field approximation with Skyrme forces are well-known [29]. Since the emphasis here is on the latent heat itself, we will only consider a few representative effective forces. We shall also briefly comment on some well known results on the phase coexistence before considering the latent heat. These will be helpful for our analysis. As a first example, we choose the Skyrme force BSk17 [46], which gives a very accurate description of the masses of nuclei all across the mass table. Four pressure isotherms calculated with BSk17 are reported in the left panel of Fig.1. These include the representative $T=0$ (solid line) and the critical $T=T_{c}$ (dashed line) isotherms. Two more isotherms, one below (dotted line) and one above (dash-dotted line) the critical one, are also displayed. The isotherms show the well known shape associated to a liquid-gas phase transition. At $T=0$, the liquid at saturation density has zero pressure and it is therefore in equilibrium with a zero density gas. As the temperature rises, the gas coexistence density shifts to finite values and the coexistence region shrinks until the critical temperature is reached. Below $T_{c}$, all isotherms present a mechanically unstable region, where the pressure decreases with density. At $T_{c}$, phase coexistence is not possible anymore and the system vaporizes completely. In terms of isotherms, one finds that, for $T>T_{c}$, the pressure becomes a monotonically increasing 
BSk17 Isotherms

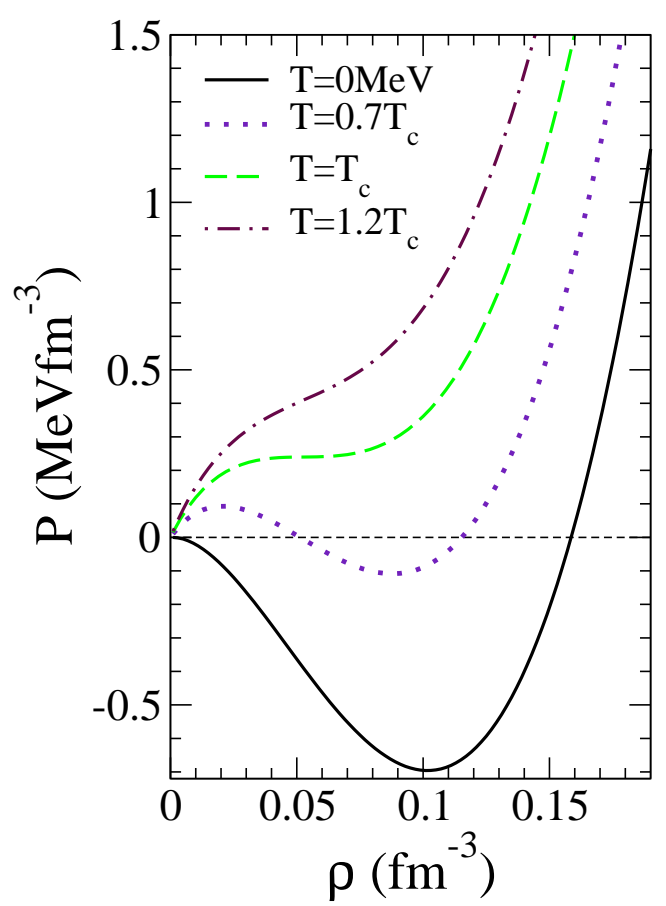

Reduced Isotherms

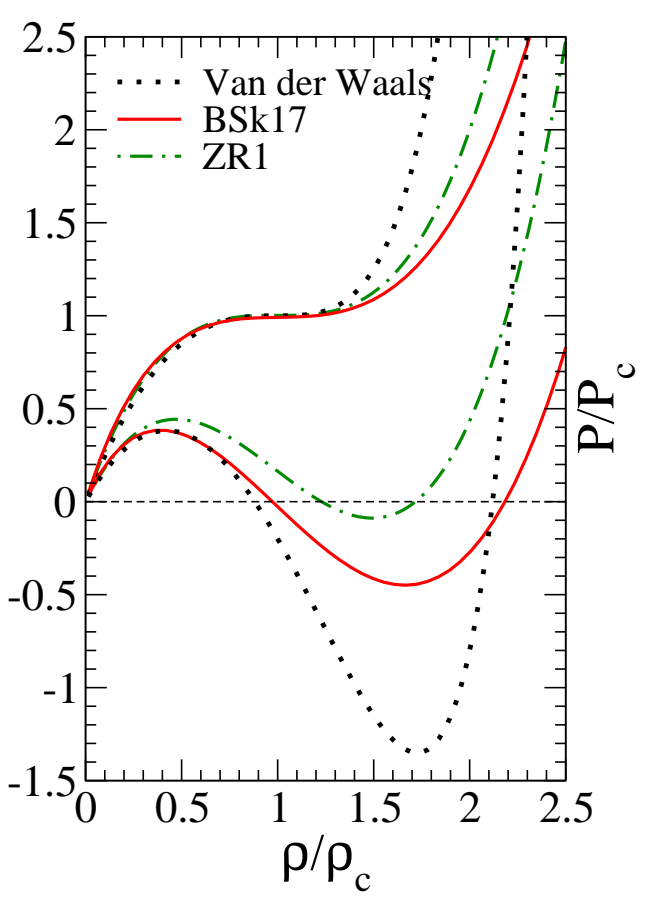

FIG. 1: (Color online) Left panel: four different isotherms for the BSk17 Skyrme force, $T_{c}=$ 15.6MeV. Right panel: reduced isotherms at $T=T_{c}$ and $T=0.7 T_{c}$ for the BSk17 (solid lines) and ZR1 (dash-dotted lines) forces. Isotherms of the Van der Waals model (dotted lines) are also shown for comparison.

function of density. For BSk17, the critical temperature turns out to be $15.6 \mathrm{MeV}$, similar to the $T_{c}$ of a wide range of modern Skyrme forces [29].

The behaviour of these isotherms is reminiscent of the Van der Waals equation of state (EoS) for real gases which, unlike the ideal gas EoS, takes into account the non-zero size of the molecules of the gas [6]. The excluded volume has a repulsive effect, in contrast to the attractive inter-molecular force. This causes the formation of regions of instability which result in a liquid-gas phase transition. To appreciate the similitudes and differences between the Van der Waals and the self-consistent mean-field EoS, we plot in the right panel of Fig. 1 the reduced isotherms $\left(P / P_{c}\right.$ vs. $\left.\rho / \rho_{c}\right)$ at $T=0.7 T_{c}$ and at $T_{c}$. In addition to the Van der Waals case, we consider the EoS obtained with BSk17 and with the ZR1 [47] forces. The latter has the highest critical temperature $\left(T_{c}=22.98 \mathrm{MeV}\right)$ among the large set of effective 
BSk17: liquid-gas phase coexistence

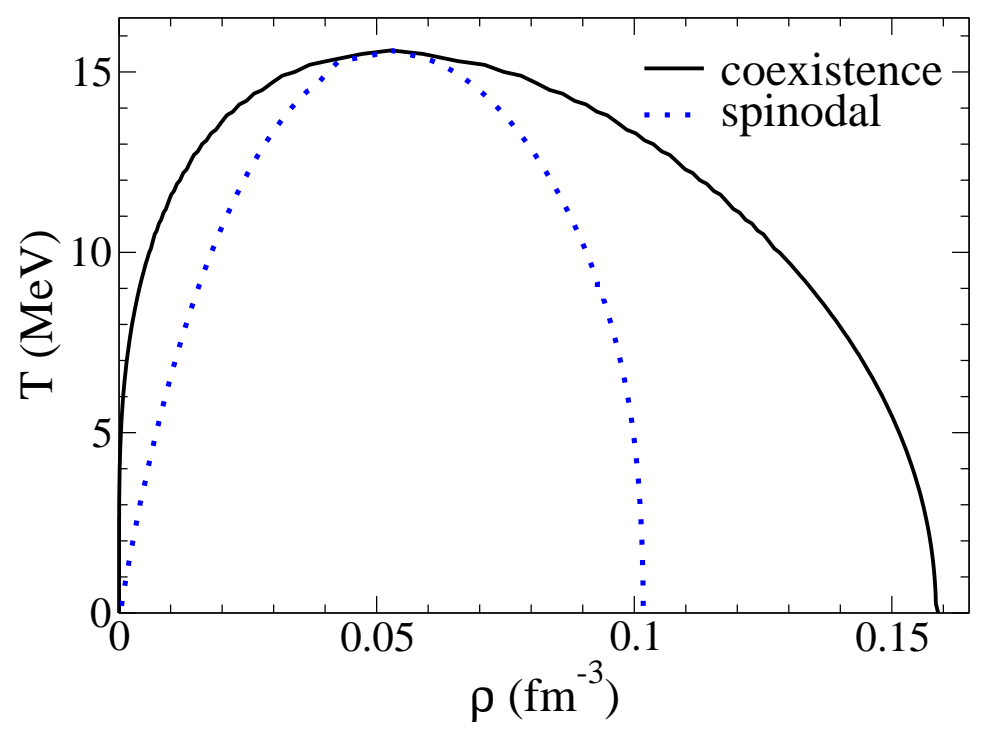

FIG. 2: (Color online) Coexistence line (solid) and spinodal line (dotted) obtained from the BSk17 force.

forces analyzed in Ref. [29].

By studying the pressure in reduced units $\left(P / P_{c}\right.$, instead of $\left.P\right)$ we expect to highlight possible resemblances between several EoS. The Van der Waals model describes the EoS of monatomic gases and liquids within a reasonable distance above and below their critical point [6]. Moreover, according to the principle of corresponding states, if we measure pressure, volume and temperature in units of $P_{c}, \rho_{c}$ and $T_{c}$, the EoS becomes universal, i.e. it is the same for a wide range of substances [6]. One might wonder whether there is something like a principle of corresponding states for EoS derived from different mean-fields. The right panel of Fig. 1 provides an insight into this matter. For $T=0.7 T_{c}$, beyond the gas phase there is a relatively important disagreement between all the reduced EoS. It is only close to the critical point that the EoS coincide. This is a consequence, as we will see later, of the fact that the Van der Waals and the self-consistent mean-field models have the same critical exponents [29].

When nuclear matter is heated up, a phase coexistence develops between a relatively high-density liquid phase and a low-density gas. In order for such equilibrium to exist, the 
chemical potential and the pressure of the two phases should be equal:

$$
\mu\left(\rho_{g}, T\right)=\mu\left(\rho_{l}, T\right), \quad P\left(\rho_{g}, T\right)=P\left(\rho_{l}, T\right) .
$$

At a given temperature, $T$, the solution of this set of equations provides a density couple, $\left(\rho_{g}, \rho_{l}\right)$, which defines the coexistence densities of the two phases. In Fig. 2, we plot the coexistence phase diagram (solid line) in the $(\rho, T)$ plane for the BSk17 force. As it has already been mentioned, at $T=0$ the liquid at saturation density is in coexistence with a zero-density gas. The gas coexistence density grows and the liquid coexistence density decreases as temperature rises. The densities of the gas and liquid phases join at the critical point, above which the liquid-gas phase coexistence disappears. Together with the coexistence line, we also plot the spinodal line (dotted line), which marks the boundary between thermodynamically stable and unstable states of matter.

The latent heat corresponding to the liquid-gas phase transition obtained with BSk17 using Eq. (10) is reported in the left panel of Fig. 3 (solid line). One can observe that the latent heat has a characteristic bump shape as a function of temperature. In the $T=0$ limit, $l$ has a finite value. For BSk17 (solid line), it grows with temperature up to a well defined maximum at $T=8.7 \mathrm{MeV}$ (with $l_{\max }=29.9 \mathrm{MeV}$ ) and then sharply goes to zero at $T=T_{c}$. To appreciate better the dependence of the latent heat of nuclear matter on the different Skyrme parameterizations, we have also performed calculations of the coexistence line and latent heat for various other forces. In addition to the already mentioned BSk17 and ZR1, we have chosen two more interactions: SLy9 [48, 49], which incorporates, by construction, the behavior of a microscopically derived EoS of neutron matter [50] and has a very low critical temperature [29]; and LNS, that reproduces the properties of BHF calculations of nuclear matter [51]. The results obtained for their latent heats are also reported in the left panel of Fig. 3. Note that all forces produce a latent heat with a similar qualitative behavior that, as we shall discuss below, can be understood from basic principles.

Let us start the discussion with the zero temperature limit of the latent heat. As mentioned previously, $l$ measures the heat that needs to be provided to the fluid to transfer a nucleon from the liquid to the gas phase. Since the equilibrium coexistence density of the gas asymptotically goes to zero when taking the $T \rightarrow 0$ limit, $l$ becomes the amount of heat needed to extract a particle from the system in that limit. In other words, for $T \rightarrow 0$, $l \rightarrow-\mu$. At the saturation density of nuclear matter, $\rho_{0}$, one actually finds $\mu=-e_{0}$ and, 
Latent heat

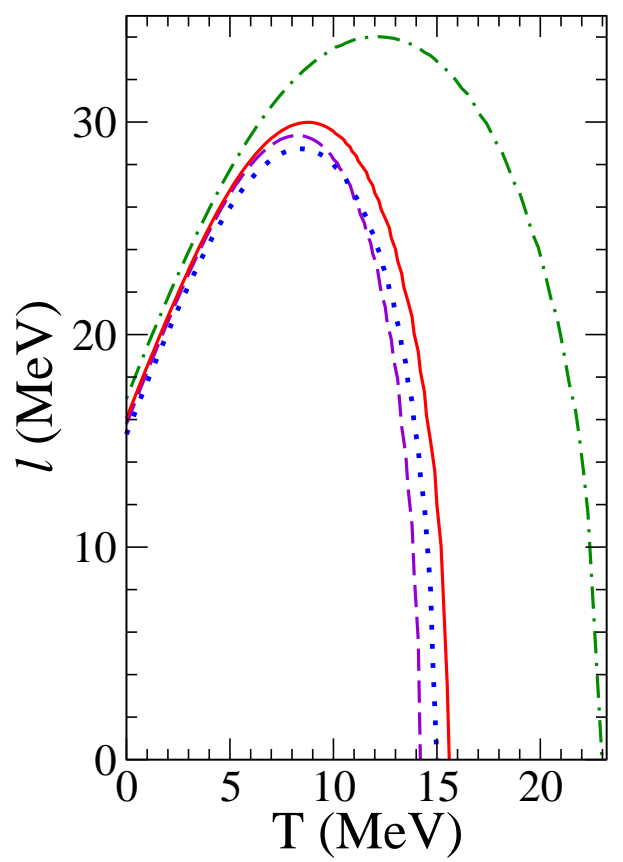

Reduced latent heat

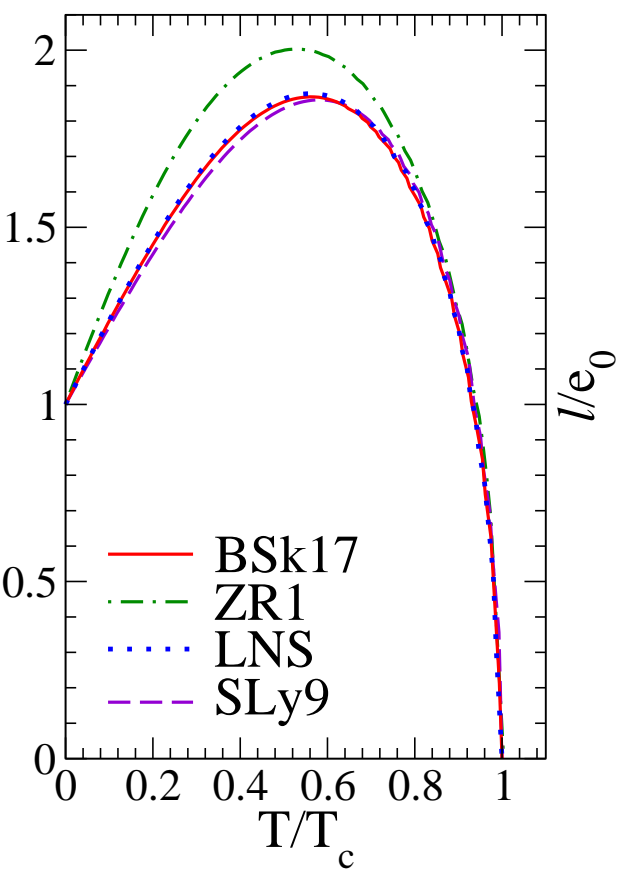

FIG. 3: (Color online) Left panel: latent heat using four different Skyrme force parametrizations. Right panel: latent heat in reduced dimensionless units using the same forces.

consequently, the latent heat is equal in absolute value to the saturation energy, $l=e_{0}$, when getting close to $T=0$. This result is independent of the interaction or the many-body approximation used to describe nuclear matter, as long as thermodynamical consistency is fulfilled. This suggests that, if one wants to obtain interaction-independent results for the latent heat, $l$ might be normalized to $e_{0}$ (see right panel of Fig. 3).

We observe that for all Skyrme parametrizations the qualitative behaviour of the latent heat is very similar (left panel of Fig. 3). The latent heat matches the value of the binding energy at $T=0$, then rises for small temperatures. The initial rise is linear and the slope seems to be independent of the Skyrme parametrization. As we shall see below, the slope of $l$ close to $T=0$ is a model-independent feature that can be understood from fundamental arguments. Further up in temperature, $l$ reaches a maximum and then drops to zero at the critical point, where the difference between the liquid and gas phases disappears. The results with this limited set of forces seem to indicate that the position and magnitude 
of the maximum in $l$ depend on the specific value of the critical temperature. Broadly speaking, higher values of $T_{c}$ shift the position and height of the maximum to larger values. This is particularly clear for the case of the ZR1 mean-field, which has the largest critical temperature. A calculation with a wider set of mean-fields, not shown here for simplicity, confirms this tendency.

A plot of the latent heat in reduced units, $l / e_{0}$, as a function of the reduced temperature, $T / T_{c}$, is presented in the right panel of Fig. 3. Similar to the right panel of Fig. 1, the reduced plot is helpful in highlighting the dependence of the latent heat on the different EoS. In general terms, we observe that the large dependence on the mean-field is eliminated to a large extent in the dimensionless plot. Close to $T=0$, the linear slope of $l$ is changed due to the fact that different mean-fields have different saturation energies, $e_{0}$. Yet, near the maximum, the reduced latent heats show a much smaller deviation compared to the absolute ones. For all Skyrme forces, the latent heat tends to peak within a limited region of temperatures, $T / T_{c} \sim 0.5-0.6$. Moreover, the peak is also quite narrowly distributed around the value $l_{\max } / e_{0} \sim 1.7-2$, which suggests that the latent heat is more determined by thermal correlations than by effective forces. Finally, as the temperature reaches the critical value, the latent heat falls to zero with a very similar temperature dependence for all forces. This identical behavior can be explained in terms of critical exponents [see Eq. (31)]. As it will be shown later, within the mean-field approximation, the critical exponents of all latent heats are the same close to the critical point.

As mentioned in the previous section, the Clausius-Clapeyron formula requires the evaluation of the derivative of the pressure with respect to the temperature along the coexistence line. The pressure along coexistence is commonly referred to as the vapor pressure. Its behavior in reduced dimensionless units, $P_{v} / P_{c}$, along the phase transition is shown in Fig. 4 for the four mentioned Skyrme parametrizations. The vapor pressure is a well behaved function of the temperature that grows from zero to $P_{c}$ as temperature increases from 0 to $T_{c}$. For low temperatures, $P_{v}$ rises very slowly and at $T=0.5 T_{c}$ it is only $10 \%$ of $P_{c}$. Above this temperature, a steady increase brings the vapor pressure very rapidly up to $P_{c}$. Note that the last portion of this increase is basically linear, in accordance to Eq. (30) below. We have checked the numerical and thermodynamical consistency between the values of the latent heat obtained with the Clausius-Clapeyron equation (Eq. (9)) and those given by the difference of entropies of the gas and the liquid phase (Eq. (10)). 


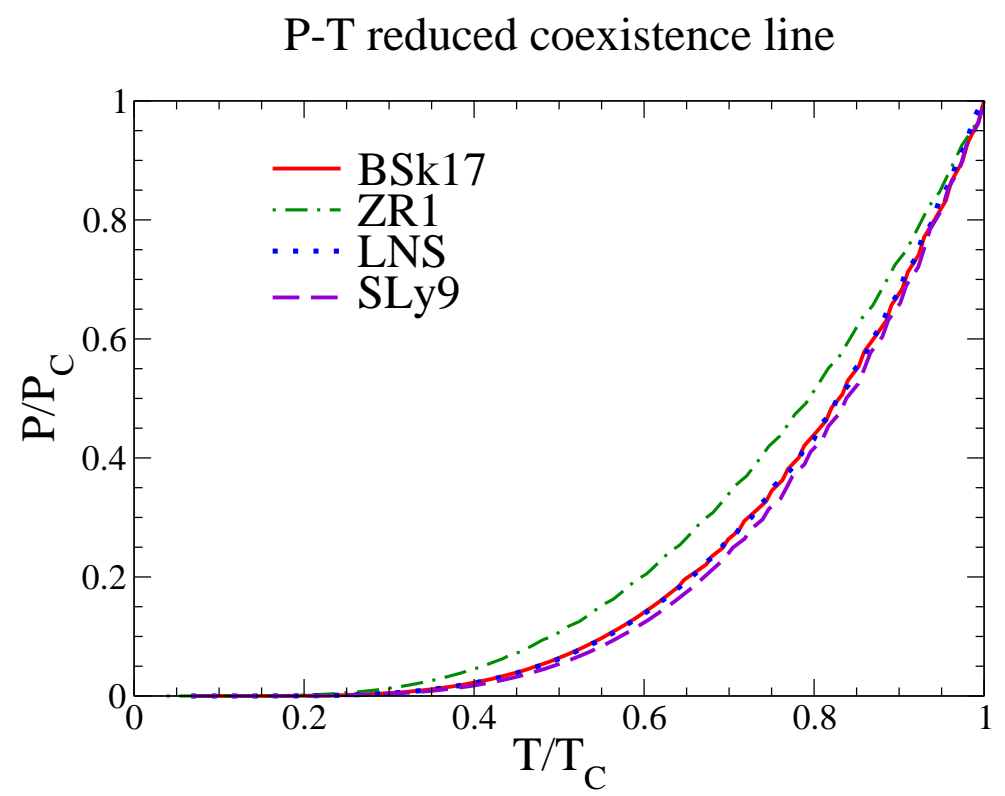

FIG. 4: (Color online) Pressure versus temperature in reduced dimensionless units obtained from the equilibrium conditions Eq. (18).

Entropy per nucleon

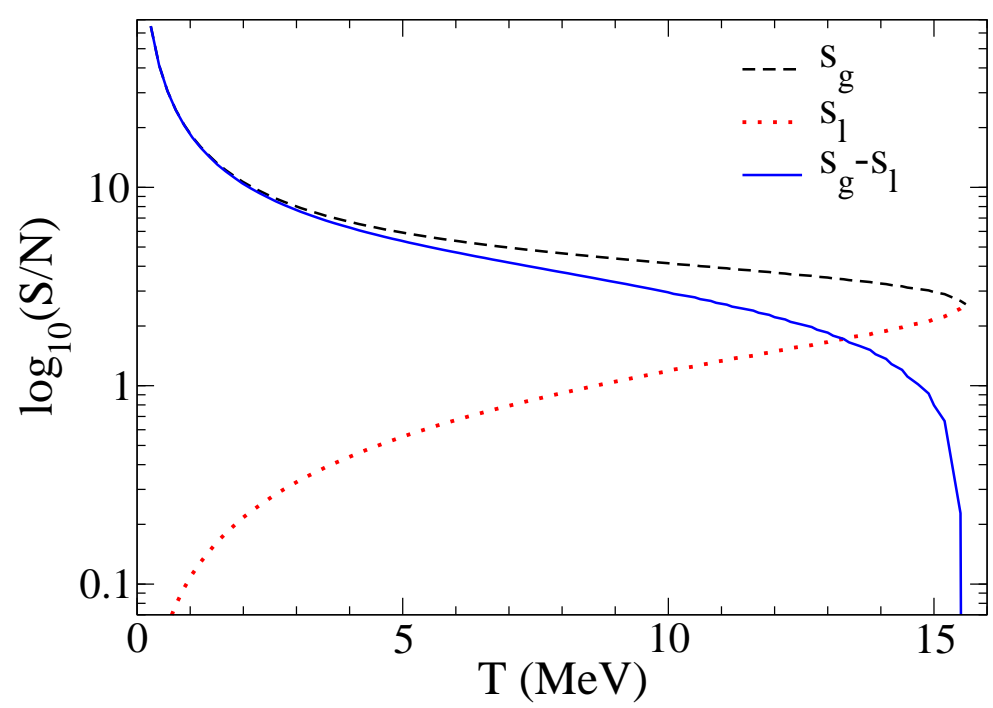

FIG. 5: (Color online) Logarithmic plot of the difference of gas and liquid entropies (solid line), entropy of the liquid (dashed line) and entropy of the gas (dotted line) along the coexistence line using the BSk17 Skyrme force. 
A basic ingredient in the evaluation of the latent heat is the difference between the entropy of the gas and that of the liquid phase [see Eq. (10)]. This difference, which has to be evaluated along the coexistence line, is shown in Fig. 5 for the BSk17 interaction. Entropies are plotted in a logarithmic scale. The difference between the entropy of the gas and that of the liquid is always a positive quantity that decreases with temperature and goes to zero as the system reaches the critical temperature. The low-temperature limit of this difference can be studied analytically and will be discussed in the next section. Note that, within this limit, the difference between the gas and liquid entropy is largely dominated by the entropy of the gas. Also notice that the variation of the liquid entropy, which should be zero at $T=0$, is much smaller than the variation of the entropy associated to the gas phase (note the logarithmic scale). While the liquid entropy along the coexistence line is an increasing function of temperature, the gas entropy is a decreasing one. Their difference, however, is dominated by the gas entropy and becomes a decreasing function of temperature. As a matter of fact, in the context of classical gases, it is customary to neglect the liquid contribution, since its entropy (or, in terms of Eq. (9), its inverse volume) is negligible with respect to the gas one [45]. In nuclear matter, this approximation would only be valid up to $T / T_{c} \sim 1 / 3$, which would lead to a maximum error in the calculation of the latent heat of about 10\%. For higher temperatures the error induced by this approximation would already reach the $30 \%$ for the maximum of the latent heat. Finally, let us stress that the maximum that appears in the latent heat at intermediate temperatures is a subtle result, arising from the product of the (linearly increasing) temperature times the (decreasing function of $T$ ) difference of entropies.

\section{ANALYTICAL LIMITS OF THE LATENT HEAT}

\section{A. Low temperature behaviour}

As we have already discussed, all Skyrme forces produce a qualitative similar behaviour of the latent heat. The value in the zero temperature limit $\left(l=e_{0}\right)$ is well understood from basic arguments. In this limit, the liquid phase is at the saturation density and therefore has zero pressure. The heat needed to transfer a nucleon from the homogeneous liquid nuclear matter phase to the zero density gas (vacuum) is just the chemical potential of the liquid 
which, at saturation, coincides with the binding energy per particle. In this subsection we will show analytically that this intuitive value is the result of a delicate balance.

In our derivation, we will not only evaluate the value of $l$ at $T=0$, but also its derivative as a function of temperature at this point. This derivative is basic in understanding the existence of a maximum in $l$. The argument goes as follows: at $T=0$, the latent heat is finite and positive. As $T \rightarrow T_{c}$, however, the latent heat must go to zero, since the liquid-gas phase transition disappears. The existence of the maximum is therefore necessarily related to the way $l$ departs from its $T=0$ value. If the slope at $T=0$ is positive, the function will first increase with $T$ and, in order to reach the zero value at $T_{c}$, at least one maximum will have to develop at some intermediate temperature. We shall also show that the slope of $l$ at $T=0$ is not only positive, but model-independent.

Let us consider the limit of low temperatures of the latent heat. As shown in Fig. 2, as $T$ approaches to zero, the dense liquid phase is in equilibrium with a very low-density gas. Due to its diluteness, we shall assume that the gas is in the classical regime and interactions are not relevant anymore. The thermodynamical properties of the gas are then given by ideal gas expressions [6] and can be computed analytically for a given gas density, $\rho_{g}$, and temperature, $T$. The pressure is simply given by

$$
P=\rho_{g} T
$$

while the gas density can be obtained in terms of its chemical potential by using the following expression:

$$
\rho_{g}=\nu\left(\frac{m}{2 \pi \hbar^{2}}\right)^{3 / 2} T^{3 / 2} e^{\mu_{g} / T} .
$$

If we substitute the previous expression in the pressure, we find the vapor pressure of nuclear matter:

$$
P_{v}(T)=\nu\left(\frac{m}{2 \pi \hbar^{2}}\right)^{3 / 2} T^{5 / 2} e^{\mu_{g} / T} .
$$

Notice that the information on the interactions in nuclear matter is contained in the chemical potential, $\mu_{g}$, which is the same for the liquid and the gas according to the equilibrium relation, Eq. (18). In the liquid branch and as $T$ approaches zero, the chemical potential tends to the energy per particle and one has $\mu_{g}=\mu_{l} \rightarrow-e_{0}$.

According to the Clausius-Clapeyron formula, Eq. (9), to calculate $l$ we need the temperature derivative of the vapor pressure along the coexistence curve. Using Eq. (21), this 
derivative becomes:

$$
\frac{d P_{v}}{d T}=\nu\left(\frac{m}{2 \pi \hbar^{2}}\right)^{3 / 2} \sqrt{T}\left[-\mu_{g}+T\left(\frac{5}{2}-\frac{\mathrm{d} \mu_{g}}{\mathrm{~d} T}\right)\right] e^{\mu_{g} / T}=\frac{\rho_{g}}{T}\left[-\mu_{g}+T\left(\frac{5}{2}-\frac{\mathrm{d} \mu_{g}}{\mathrm{~d} T}\right)\right] .
$$

All temperature derivatives are to be taken as derivatives along the coexistence line. Because of the equilibrium condition in Eq. (18), the chemical potential can be computed from the liquid one which, close to saturation, should be that of a degenerate Fermi gas. The Sommerfeld expansion then guarantees that the temperature dependence of $\mu_{l}$ is quadratic in $T$ [52]. Consequently, the temperature derivative in the last term $\frac{\mathrm{d} \mu_{g}}{\mathrm{~d} T} \sim \mathcal{O}(T)$ and thus can be neglected in the following considerations. Note that the low temperature limit of the previous expression, Eq. (22), is zero. However, when the latent heat is considered in the zero temperature limit, we need to explicitly take into account the prefactors, whose cancelation leads to:

$$
\lim _{T \rightarrow 0} l(T)=\lim _{T \rightarrow 0} T\left(\frac{1}{\rho_{g}}-\frac{1}{\rho_{l}}\right) \frac{d P_{v}}{d T}=\lim _{T \rightarrow 0} T \frac{1}{\rho_{g}} \frac{d P_{v}}{d T}=-\mu_{g}=e_{0} .
$$

When taking the limit, we have considered that the term containing the liquid density goes to zero because the gas density present in the derivative of the vapor pressure (see Eq. (22)) goes to zero in this limit, and that the linear terms in temperature within the brackets in Eq. (22) are subleading.

Let us now compute the derivative of $l$ close to zero temperature:

$$
\frac{d l}{d T}=\left(\frac{1}{\rho_{g}}-\frac{1}{\rho_{l}}\right) \frac{d P_{v}}{d T}+T\left(\frac{1}{\rho_{g}}-\frac{1}{\rho_{l}}\right) \frac{d^{2} P_{v}}{d T^{2}}+T \frac{d P_{v}}{d T} \frac{d}{d T}\left(\frac{1}{\rho_{g}}-\frac{1}{\rho_{l}}\right) .
$$

The $T \rightarrow 0$ limit of the first term is obtained from Eq. (22). The second term involves the second derivative of the vapor pressure, which is also easily computed from Eq. (22) and yields:

$$
T\left(\frac{1}{\rho_{g}}-\frac{1}{\rho_{l}}\right) \frac{d^{2} P_{v}}{d T^{2}} \sim-\frac{1}{T^{2}}\left(\mu^{2}-4 \mu T+\frac{15}{4} T^{2}\right) .
$$

The third term involves derivatives of the gas density, Eq. (20), while the liquid density is neglected:

$$
T \frac{d P_{v}}{d T} \frac{d}{d T}\left(\frac{1}{\rho_{g}}-\frac{1}{\rho_{l}}\right) \sim \frac{1}{T^{2}}\left(\mu^{2}-3 \mu T+\frac{15}{4} T^{2}\right) .
$$

Collecting the different contributions, one gets :

$$
\lim _{T \rightarrow 0} \frac{d l}{d T}=\left[\frac{5}{2}-\frac{\mu}{T}\right]+\left[\frac{\mu^{2}}{T^{2}}-\frac{3 \mu}{T}+\frac{15}{4}\right]-\left[\frac{\mu^{2}}{T^{2}}-\frac{4 \mu}{T}+\frac{15}{4}\right]=\frac{5}{2}
$$




\section{Latent heat fit at low $\mathrm{T}$}

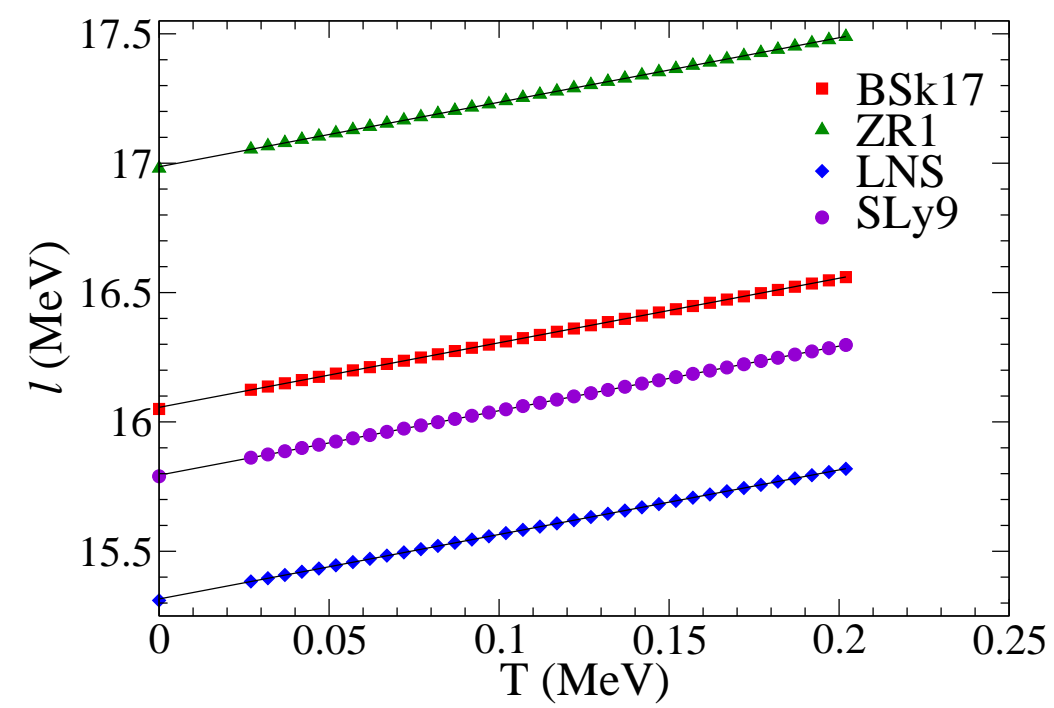

FIG. 6: (Color online) Latent heat versus temperature for very low $T$. Symbols represent selfconsistent mean-field results and lines their respective linear regression fits.

The derivative of the latent heat with respect to the temperature in the limit $T \rightarrow 0$ is therefore independent of the interaction. Moreover, it is positive and equal to $5 / 2$.

In Fig. 6 we show a numerical proof of Eq. (27). For the different Skyrme force parametrizations considered in Fig. 3, we focus on the low-temperature behaviour of the latent heat (symbols). The slopes for the linear regression fits (lines) of these numerical data are in very good agreement with the value 5/2 up to two significant digits.

This result is not only valid regardless of the effective interaction, but it is also valid no matter which many-body approximation is considered. The only assumption that has been made is that the gas equilibrium density enters the classical regime as the temperature decreases. Consequently, one should get the same result in approaches that go beyond the $\mathrm{HF}$ approximation. It is also important to note that this result is independent of the system under study and therefore should be generically valid for the liquid-gas phase transition of any extended normal fermionic system. As discussed previously, the positiveness of this derivative necessarily implies that a maximum in the latent heat must develop. As a result, we expect a maximum in the latent heat of any fermionic system that presents a liquid-gas phase transition. 


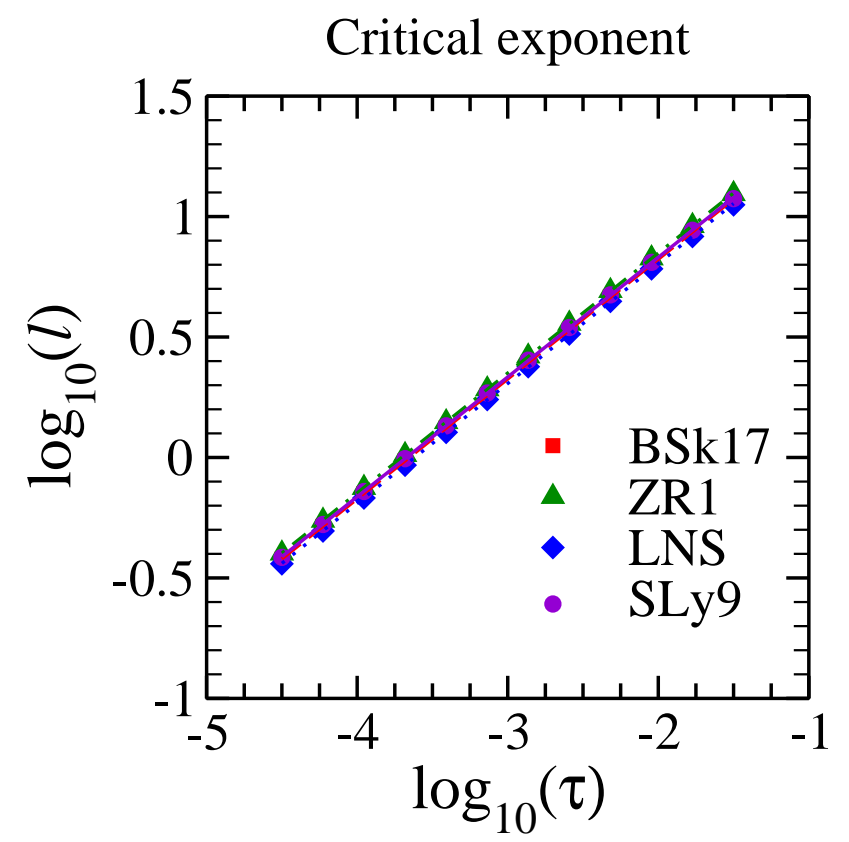

FIG. 7: (Color online) Logarithmic plot of the latent heat versus the reduced temperature. The slope of the different curves gives the critical exponent. Symbols represent self-consistent mean-field results and lines their respective linear regression fits.

\section{B. Critical behaviour}

Critical exponents characterize the properties of phase transitions [6, 45, 53]. Close to the critical point, all the thermodynamical properties can be described in terms of a handful of exponents. Their knowledge facilitates the understanding of the properties of systems close to criticality. The latent heat is not an exception and it can also be described, close to the critical point, in terms of a critical exponent. As shown in Ref. [45], the critical exponent for $l$ is the same as that of the order parameter.

This result can be understood in terms of the mean-field theory of fluctuations as follows. Consider the reduced pressure, $\pi=\left(P-P_{c}\right) / P_{c}$, close to the critical point. Expanding to first order in the reduced temperature, $\tau=\left(T-T_{c}\right) / T_{c}$, and to third order in the reduced density, $\eta=\left(\rho-\rho_{c}\right) / \rho_{c}$, one finds:

$$
\pi=a \tau+b \tau \eta+c \eta^{3}+c^{\prime} \tau \eta^{2}
$$


Note that the conditions to find the critical point,

$$
\frac{\partial \pi}{\partial \eta}=\frac{\partial^{2} \pi}{\partial \eta^{2}}=0
$$

have already been used to eliminate possible explicit linear and quadratic terms in $\eta$. To find the pressure at phase coexistence, one can use Eq. (18) in Eq. (28) to find the symmetric gas and liquid coexistence points. One can then show that the coexistence pressure is given by:

$$
\left.\pi\right|_{\text {coex }}=a \tau \text {. }
$$

Using the Clausius-Clapeyron relation, the latent heat close to the critical point becomes:

$$
l=\frac{a P_{c}}{2 \rho_{c}}\left(\rho_{l}-\rho_{g}\right)(1+\tau) \sim(-\tau)^{\beta} .
$$

In this expression, we have considered that the difference in densities is the order parameter of the phase transition and that the latter is governed by the $\beta$ critical exponent, $\rho_{l}-\rho_{g} \sim$ $(-\tau)^{\beta}$. As any mean-field theory [6, 29], self-consistent Hartree-Fock calculations of the nuclear matter liquid-gas phase transition yield a critical exponent $\beta=1 / 2$.

We have checked numerically that the critical exponent of $l$ is indeed $\beta=1 / 2$. Fig. 7 shows a logarithmic plot of $l$ versus the reduced critical temperature (symbols). Linear regression fits have been performed and are shown with lines. The correlation coefficients are close to 1 to within at least four digits in all cases. Such a linear behavior of the data confirms the scaling of $l$ with $\tau$. The slopes of these lines have also been extracted and agreement with the $\beta$ derived from the coexistence line is good up to the third digit. To our knowledge, this is the first time that the critical exponent of the latent heat for nuclear matter is computed and that its equivalence to $\beta$ is confirmed numerically in the framework of a Hartree-Fock mean-field theory. However, one should keep in mind the limitations of the mean-field theory for the calculation of the critical exponents.

\section{SUMMARY AND CONCLUSIONS}

In this work, we have have analyzed in detail the latent heat of the liquid-gas phase transition of symmetric nuclear matter. The latent heat describes the amount of heat needed to transfer one nucleon from the liquid to the gas phase, and it can be used as 
a further characterization of the phase transition. We have been motivated by experimental results which suggest that, in the phase transition occurring in nuclear multifragmentation collisions, a latent heat of around $l \sim 2-8 \mathrm{MeV}$ can be observed [4, 15, 19].

We have used self-consistent Hartree-Fock calculations at finite temperature to obtain numerical results. Four different characteristic mean-fields have been used to determine the inherent mean-field dependence of the results. The qualitative behavior of the latent heat as a function of temperature is very similar for all effective interactions. Within the HartreeFock approximation, the latent heats derived from different mean-field parametrizations fall within a narrow band when the temperature is scaled by $T_{c}$ and $l$ is scaled by $e_{0}$.

In the $T \rightarrow 0$ limit, the latent heat coincides with the binding energy per particle at saturation density. The latent heat can also be computed from the difference in entropies between the gas and the liquid phases. We have seen that the gas entropy dominates over the liquid one in a wide range of temperatures. At finite but low temperatures, the latent heat rises linearly with temperature. A careful analysis shows that the slope of this linear trend is $5 / 2$, regardless of the mean-field parametrization or the many-body approximation. We have confirmed this trend with numerical finite temperature HF calculations. This modelindependent result is valid for all normal fermionic systems. To our knowledge, it is the first time that this result is discussed.

Knowing that a) $l$ is positive and finite at $T=0$, b) it has a positive slope near $T=0$ and c) it goes to zero at $T=T_{c}$, necessarily implies the existence of a maximum in the latent heat. Mean-field numerical calculations suggest that this maximum is located around $T \sim 0.6 T_{c}$ and that $l_{\max } \sim 1.8 e_{0}$. While the exact position of the well-defined maximum can depend on the effective force or on the many-body approach used to describe nuclear matter, its existence is guaranteed independently of the mean-field parametrization or the many-body approximation. Moreover, this result should be valid for any normal fermionic system that presents a liquid-gas phase transition.

It is not particularly easy to understand physically why the latent heat should present a maximum as a function of temperature. One might interpret the presence of this maximum as a manifestation of the underlying NN interaction. At low temperatures, the latent heat rises because more work is needed to break the attractive part of the strong interaction when transferring a nucleon from the more structured liquid to the gas phase. At higher temperatures, the thermal motion of the particles would provide most of the work and thus 
less latent heat is needed to break the bonding between particles in the liquid. Here, we have given an analytical demonstration of the existence of the peak.

Concerning the behavior of the latent heat near the critical point, we have shown numerically that it can be described in terms of a single critical exponent. As predicted by the theory of phase transitions, this critical exponent is the same as the one associated to the order parameter. In the case of Hartree-Fock calculations in nuclear matter, $\beta=1 / 2$, and there is a very good numerical agreement between both exponents. All in all, the latent heat can be characterized in the low, intermediate and (close to) critical temperature regimes from very basic principles. It is precisely this generic nature which might motivate the use of the latent heat as a tool in analyzing the liquid-gas phase transition in other normal fermionic systems.

We are well aware that a connection between experimental observations of multifragmentation collisions and theoretical results of homogenous nuclear matter is not at all transparent. Finite size effects play a capital role in determining the thermodynamical properties of nuclei and the latent heat is not an exception [36]. Nuclear matter values at its maximum suggest that the latent heat is up to 10 times higher than that extracted from different experimental analysis $[15,16,19]$. Even at the theoretical level, it is not clear how to define a liquid and a gas phase in a self-confined system [9]. Nevertheless, one might naively expect that the appearance of a maximum in the latent heat might have a substantial suppression effect on the yields of light particles. We hope that the present study, where we have highlighted model-independent and basic arguments for the latent heat, will encourage further experimental analysis of the latent heat in multifragmentation collisions.

\section{ACKNOWLEDGEMENTS}

This work has been supported by Grants No. FIS2008-01661 (Spain), and No. 2009SGR1289 from Generalitat de Catalunya, a Marie Curie Intra European Fellowship within the $7^{\text {th }}$ Framework programme and STFC grant ST/F012012, FCT (Portugal) under project CERN/FP/109316/2009, and by COMPSTAR, an ESF Research Networking Programme. 
The authors are very grateful to professor A. Planes for useful and stimulating discussions.

[1] P. J. Siemens, Nature 305, 410 (1983).

[2] S. D. Gupta, A. Z. Mekjian, and M. B. Tsang, Advances in Nuclear Physics, Vol. 26 (Springer US, 2006), chap. Liquid-gas phase transition in nuclear multifragmentation, p. 89.

[3] B. Borderie and M. F. Rivet, Prog. Part. Nucl. Phys. 61, 551 (2008).

[4] J. Pochodzalla, Prog. Part. Nucl. Phys. 39, 443 (1997).

[5] P. Ring and P. Schuck, The Nuclear Many-Body Problem (Springer, 1984), 1st ed.

[6] K. Huang, Statistical Mechanics (John Wiley and Sons, 1987), 2nd ed.

[7] J. A. Hauger et al., Phys. Rev. Lett. 77, 235 (1996).

[8] V. E. Viola, K. Kwiatkowski, and W. A. Friedman, Phys. Rev. C 59, 2660 (1999).

[9] P. Bonche, S. Levit, and D. Vautherin, Nucl. Phys. A 427, 278 (1984).

[10] J. B. Natowitz et al., Phys. Rev. C 52, 2322 (1995).

[11] V. Sefling et al., Phys. Rev. Lett. 80, 3928 (1998).

[12] X. Campi, Phys. Lett. B 208, 351 (1988).

[13] M. L. Gilkes et al., Phys. Rev. Lett. 73, 1590 (1994).

[14] J. B. Elliott et al., Phys. Rev. Lett. 88, 042701 (2002).

[15] M. d'Agostino et al., Phys. Lett. B 473, 219 (2000).

[16] J. Pochodzalla et al., Phys. Rev. Lett. 75, 1040 (1995).

[17] J. B. Natowitz, R. Wada, K. Hagel, T. Keutgen, M. Murray, A. Makeev, L. Qin, P. Smith, and C. Hamilton, Phys. Rev. C 65, 034618 (2002).

[18] B. Pichon et al., Nucl. Phys. A 779, 267 (2008).

[19] E. Bonnet et al., Phys. Rev. Lett. 103, 072701 (2009).

[20] B. terHaar and R. Malfliet, Phys. Rev. Lett. 56, 1237 (1986).

[21] M. Baldo and L. S. Ferreira, Phys. Rev. C 59, 682 (1999).

[22] A. Rios, A. Polls, A. Ramos, and H. Müther, Phys. Rev. C 78, 044314 (2008).

[23] V. Somà and P. Bożek, Phys. Rev. C 80, 025803 (2009).

[24] A. Mukherjee and V. R. Pandharipande, Phys. Rev. C 75, 035802 (2007).

[25] H. Müller and B. D. Serot, Phys. Rev. C 52, 2072 (1995).

[26] D. Vautherin, Advances in Nuclear Physics, Vol. 22 (Springer US, 1996), chap. Many-body 
methods at finite temperature, p. 123.

[27] T. Sil, S. K. Samaddar, J. N. De, and S. Shlomo, Phys. Rev. C 69, 014602 (2004).

[28] M. Bender, P.-H. Heenen, and P.-G. Reinhard, Rev. Mod. Phys. 75, 121 (2003).

[29] A. Rios, Nucl. Phys. A 845, 58 (2010).

[30] C. Horowitz and A. Schwenk, Nucl. Phys. A 776, 55 (2006).

[31] A. L. Goodman, J. I. Kapusta, and A. Z. Mekjian, Phys. Rev. C 30, 851 (1984).

[32] M. Beyer, S. A. Sofianos, C. Kuhrts, G. Röpke, and P. Schuck, Phys. Lett. B 488, 247 (2000).

[33] S. Typel, G. Röpke, T. Klähn, D. Blaschke, and H. H. Wolter, Phys. Rev. C 81, 015803 (2010).

[34] S. S. Avancini, C. C. Barros Jr., D. P. Menezes, and C. Providência, Phys. Rev. C 82, 025808 (2010).

[35] F. Gulminelli, P. Chomaz, A. H. Raduta, and A. R. Raduta, Phys. Rev. Lett. 91, 202701 (2003).

[36] S. J. Lee and A. Z. Mekjian, Phys. Rev. C 56, 2621 (1997).

[37] X. Campi, H. Krivine, and E. Plagnol, Phys. Lett. B 385, 1 (1996).

[38] L. G. Sobotka, R. J. Charity, J. Toke, and W. U. Schröder, Phys. Rev. Lett. 93, 132702 (2004).

[39] D. V. Shetty, G. A. Souliotis, S. Galanopoulos, and S. J. Yennello, Phys. Rev. C 79, 034603 (2009).

[40] J. Randrup, Phys. Rev. C 79, 054911 (2009).

[41] T. H. R. Skyrme, Philos. Mag. 1, 1043 (1956).

[42] T. H. R. Skyrme, Nucl. Phys. 9, 615 (1959).

[43] J. Rikovska Stone, J. C. Miller, R. Koncewicz, P. D. Stevenson, and M. R. Strayer, Phys. Rev. C 68, 034324 (2003).

[44] U. Gupta and A. K. Rajagopal, Phys. Rev. A 21, 2064 (1980).

[45] L. D. Landau and E. M. Lifshitz, Statistical Mechanics, Part 1 (Butterworth-Heineman, 1980), 3rd ed.

[46] S. Goriely, N. Chamel, and J. M. Pearson, Phys. Rev. Lett. 102, 152503 (2009).

[47] H. Jaqaman, A. Z. Mekjian, and L. Zamick, Phys. Rev. C 29, 2067 (1984).

[48] E. Chabanat, Ph.D. thesis, University of Lyon (1995).

[49] E. Chabanat, P. Bonche, P. Haensel, J. Meyer, and R. Schaeffer, Nucl. Phys. A 627, 710 (1997). 
[50] R. B. Wiringa, V. Fiks, and A. Fabrocini, Phys. Rev. C 38, 1010 (1988).

[51] L. G. Cao, U. Lombardo, C. W. Shen, and N. Van Giai, Phys. Rev. C 73, 014313 (2006).

[52] N. W. Ashcroft and N. D. Mermin, Solid State Physics (Holt, Rinehart and Winston, 1976).

[53] L. P. Kadanoff et al., Rev. Mod. Phys. 39, 395 (1967). 